\title{
How to Achieve High Encapsulation Efficiencies for Macromolecular and Sensitive APIs in Liposomes
}

\author{
Kirsten Ullmann ${ }^{1, *(D)}$, Gero Leneweit ${ }^{2,3}$ LD $^{(D)}$ and Hermann Nirschl ${ }^{1}$ \\ 1 Institute of Mechanical Process Engineering and Mechanics, Process Machines, Karlsruhe Institute of \\ Technology (KIT), 76131 Karlsruhe, Germany; hermann.nirschl@kit.edu \\ 2 Carl Gustav Carus-Institute, Association for the Promotion of Cancer Therapy, \\ 75223 Niefern-Oeschelbronn, Germany; gero.leneweit@carus-institut.de \\ 3 Abnoba $\mathrm{GmbH}, 75223$ Niefern-Oeschelbronn, Germany \\ * Correspondence: kirsten.ullmann@kit.edu; Tel.: +49-721-60842429
}

Citation: Ullmann, K.; Leneweit, G.; Nirschl, H. How to Achieve High Encapsulation Efficiencies for Macromolecular and Sensitive APIs in Liposomes. Pharmaceutics 2021, 13, 691. https://doi.org/10.3390/ pharmaceutics 13050691

Academic Editor: Thierry Vandamme

Received: 16 April 2021

Accepted: 7 May 2021

Published: 11 May 2021

Publisher's Note: MDPI stays neutral with regard to jurisdictional claims in published maps and institutional affiliations.

Copyright: (c) 2021 by the authors. Licensee MDPI, Basel, Switzerland. This article is an open access article distributed under the terms and conditions of the Creative Commons Attribution (CC BY) license (https:// creativecommons.org/licenses/by/ $4.0 /)$.

\begin{abstract}
This research highlights the capacity of a newly introduced centrifugation process to form liposomes from water-in-fluorocarbon nano-emulsions stabilized with phospholipids to incorporate macromolecular and sensitive active pharmaceutical ingredients (API). The encapsulation efficiency of the produced liposomes, incorporating fluorescein-sodium, bovine serum albumin and fluorecein isothiocyanate dextran as model APIs, is determined by applying Vivaspin ${ }^{\circledR}$ centrifugation filtration and quantified by UV-Vis spectroscopy. It was found that higher densities of the fluorocarbons used as the hydrophobic phase enable a higher encapsulation efficiency and that an efficiency of up to $98 \%$ is possible depending on the used phospholipid. Among the engineering aspects of the process, a comparison between different membrane substances was performed. Efficiency increases with a higher phospholipid concentration but decreases with the addition of cholesterol. Due to the higher bending modulus, liposome formation is slowed down by cholesterol during liposome closure leading to a greater leakage of the model API. The encapsulation of bovine serum albumin and dextran, both investigated under different osmotic conditions, shows that an efflux negatively affects the encapsulation efficiency while an influx increases the stability. Overall, the process shows the potential for a very high encapsulation efficiency for macromolecules and future pharmaceutical applications.
\end{abstract}

Keywords: encapsulation efficiency; liposomes; phospholipids; fluorocarbon; nano-emulsions; active pharmaceutical ingredients

\section{Introduction}

To this day, cancer remains one of the most severe diseases, even though mortality has decreased overall due to steady reductions in smoking and advances in early detection and treatments for patients [1]. However, some death rates increased from 2012-2016, such as for cancers of the liver, pancreas, uterus, brain and nervous system [1]. Common methods in cancer therapy, such as the use of chemotherapeutic substances, are not specific to cancer cells but affect healthy cells as well and harm the patient to a large extent. A way to prevent the unnecessary harm of the human body is the specific targeting of the tumour by using, for example, fusion protein engineered antibodies [2]. These approaches still face the problem of degradation because the endogenous defence detects the antibodies that carry the drug as an intruder. A different approach is the encapsulation of pharmaceutical ingredients in liposomes which can be delivered to the target cell without previous degradation [3]. Liposomes consist of phospholipids that play a key role in the food and pharma industry because of their ubiquity in all organisms and their absolute safety [4]. They are often utilized as natural emulsifiers. Using PLs as emulsifiers or building blocks, they contribute to the properties of the delivery system. The amphiphilic characteristics of the phospholipids are the constitutive base of all biological membranes 
and allow the carrying of an active ingredient inside, while transport through the blood circulation is possible without provoking an immediate immune response [4].

Manufacturing methods for liposomes and other drug delivery vehicles have been the subject of intensive research for over 30 years. A conventional laboratory method for the production of liposomes is the film method, in which the phospholipids, possibly with a hydrophobic active ingredient, are dissolved in a solvent, e.g., ethanol or a mixture of methanol and chloroform. The solvent is then removed in the rotary evaporator so that the lipids form ordered bilayers at the wall of the flask. The hydrophilic active ingredient is encapsulated by adding the aqueous solvent. The lipids start swelling and form a heterogeneous suspension of multilamellar vesicles (liposomes) in which the active ingredient is encapsulated. Depending on the solubility of the active ingredient, the continuous phase can be aqueous or hydrophobic $[5,6]$.

The production of uni-lamellar liposomes with defined sizes can be achieved in different ways. One possibility is the extrusion process, in which the solution is forced through nano-meter sized pores in a membrane under high pressure. The multilayers are fractured as they enter the nanopores and multilamellar vesicles are converted into uni-lamellar liposomes [7]. The membrane pore size controls the size of the resulting liposomes [8]. Another alternative for large production volumes is the use of a homogenizer [9]. The liposome suspension passes through the homogenizer several times and the liposomes decrease in size with each pass. The minimally achievable sizes are $20 \mathrm{~nm}[10,11]$.

Based on their mechanism of action all the above methods are reliable regarding size mono-dispersity, but the concentration of active ingredients inside is the same as outside at the moment of vesicle closure. The encapsulation efficiency found in the literature for high molecular weight molecules is only in the low double-digit range and varies from about $2-50 \%$ in cases of affinity between active ingredients and liposomal membrane [12,13]. Higher efficiencies have not yet been established as so-called 'remote loading' is only feasible for small molecules that diffuse through phospholipid bilayers, but not for macromolecular APIs. A "high" encapsulation efficiency is proposed by $\mathrm{Xu}$ et al. who use freeze-thaw cycles for entrapment of proteins [14]. Yet the proposed method does not exceed the 50\% level either. Furthermore, the use of solvents is necessary for the majority of the manufacturing methods mentioned. However, even small traces of solvents are undesirable for application as a carrier for active ingredients in the pharmaceutical or food sector as they destabilize and degrade many active ingredients, especially peptides or proteins. A comprehensive removal of possible solvent residues in the production process is therefore extremely costly and time-consuming as is the removal of the API which is not encapsulated and thus, found freely in the solution, needing additional separation and purification steps [15].

Engineering methods, however, are not common even though they have a great potential for large scale application. Pautot et al. were the first to introduce a centrifugation method where the droplets of a water-in-oil $(w / o)$ nano-emulsion transfer to a second aqueous phase to create a bilayer [16]. While this method works for large uni-lamellar vesicles of between 1 and $10 \mu \mathrm{m}$, several limitations exist such as an appropriate size for pharmaceutical applications, the stability of nano-emulsions, and oranogel formation between the three phases (water, oil and phospholipids). A smaller size of liposome was achieved by de Matos et al. [17]. However, the phase transfer and encapsulation efficiencies were still insufficient. Previous research tackled the above problems and revealed that several advantages regarding the centrifugation process were achieved by using a fluorocarbon as hydrophobic phase instead of a hydrocarbon (such as squalene or dodecane, used previously); hindrance by interfacial tension during phase transfer is compensated for by the much stronger density difference between the hydrophobic and aqueous phase $\left(\Delta \varrho \approx 1 \mathrm{~g} / \mathrm{cm}^{3} ; \varrho_{\text {water }}=0.998 \mathrm{~g} / \mathrm{cm}^{3} ; \varrho_{\text {fluorocarbon }}=2.03 \mathrm{~g} / \mathrm{cm}^{3}\right)$, enabling transfer. Organogel formation is no longer detectable. Nano-emulsion droplets are stable for several weeks and liposome production is successful [18]. Because of the 
heavier hydrophobic phase, the aqueous droplets float up instead of sedimenting during centrifugation, which enables easy removal of the liposomal suspension from the top.

The question remains as to whether a high encapsulation efficiency is possible by applying the centrifugation process. While De Matos et al. used the centrifugation method to produce asymmetric liposomes and encapsulated nucleic acids, they used squalene for the preparation of nano-emulsions leading to a plasmid encapsulation of 10-15\% [17]. Hence, an evaluation of the encapsulation efficiency of the formed liposomes from the water-in-fluorocarbon $(\mathrm{w} / \mathrm{fc})$ nano-emulsion remains to be carried out.

The aim of this work is to show the capacity of the process for high encapsulation efficiency by using different fluorocarbons and the phospholipids DPPC, DPPG and DMPC, evaluated with fluorescein-sodium (FS) as a low molecular weight hydrophilic marker, as well as bovine serum albumin (BSA) and fluorescently labelled dextran as high molecular weight protein and polymer, respectively. The sequence of different analytical detection methods was evaluated for its capacity to determine the amount of the encapsulated model API, while being able to trace not only the API surrogates itself but also other compounds such as phospholipids and the fluorocarbon phase.

\section{Materials and Methods}

\subsection{Materials}

Phospholipids utilized for all experiments were provided by Lipoid (Ludwigshafen, Germany). The synthetic phospholipids 1,2-dimyristoyl-sn-glycero-3-phostphatidylcholine (DMPC), 1,2-dipalmitoyl-sn-glycero-3-phosphatidylcholine (DPPC) and 1,2-dipalmitoyl-snglycero-3-phospho-rac-glycerol (DPPG) were received in powder form. Cholesterol (chol.) was purchased from Carl Roth (Karlsruhe, Germany). Perfluoro-perhydro-phenanthrene $\left(\mathrm{C}_{14} \mathrm{~F}_{24}\right)$ was purchased from F2 Chemicals (Preston, UK) and has a density of $2.03 \mathrm{~g} / \mathrm{cm}^{3}$ and a refractive index (RI) of 1.331. For comparison, the fluorocarbons perfluoro-heptane $\left(\mathrm{C}_{7} \mathrm{~F}_{16}, \mathrm{RI}=1.26, \varrho=1.72 \mathrm{~g} / \mathrm{cm}^{3}\right)$, perfluoro-1,3-dimethylcyclohexane $\left(\mathrm{C}_{8} \mathrm{~F}_{16}, \mathrm{RI}=1.2895\right.$, $\left.\varrho=1.83 \mathrm{~g} / \mathrm{cm}^{3}\right)$ and perfluoro-methyl-decalin $\left(\mathrm{C}_{11} \mathrm{~F}_{20}, \mathrm{RI}=1.3195, \varrho=1.92 \mathrm{~g} / \mathrm{cm}^{3}\right)$ from F2 Chemicals were also tested. Phosphate buffer consisted of a 1:4.2 mixture of sodium di-hydrogen phosphate $\left(\mathrm{NaH}_{2} \mathrm{PO}_{4}\right)$ and di-sodium hydrogen phosphate $\left(\mathrm{Na}_{2} \mathrm{HPO}_{4}\right.$, both Carl Roth, Karlsruhe, Germany). Encapsulation efficiency was measured via fluoresceinsodium (FS, Carl Roth, Karlsruhe, Germany), BSA (VWR International GmbH, Darmstadt, Deutschland) and fluorescein isothiocyanate-dextran (FITC-D, $\mathrm{M}_{\mathrm{W}}=70 \mathrm{kDa}$, Merck, Darmstadt, Germany). For phospholipid quantification, perchloric acid (70\%) was purchased from Carl Roth (Karlsruhe, Germany). Further chemicals used for the phosphorus assay were ascorbic acid, hexa-ammonium molybdate and sodium dihydrogen phosphate as a standard phosphate solution (all Carl Roth, Karlsruhe, Germany).

\subsection{Preparation of Aqueous Lipid Stock Suspension}

Lipid suspensions were prepared with different concentrations of phospholipids in $1 \mathrm{~mL}$ with $15 \mathrm{mM}$ phosphate buffer if not stated otherwise. The preparation was performed in micro reaction tubes (Eppendorf, Hamburg, Germany). Ultrasound provided by a $3 \mathrm{~mm}$ sonotrode tip (Digital Sonifier 450, Branson Ultrasonic, Danbury, CT, USA) was used for the dispersion of phospholipids with a $100 \%$ cycle and an amplitude of $10 \%$ for $10 \mathrm{~s}$ followed by a $50 \%$ cycle and $10 \%$ amplitude for $10 \mathrm{~min}$. The temperature was kept constant at $30{ }^{\circ} \mathrm{C}$. To allow the determination of the encapsulation efficiency, fluorescein-sodium was added to the stock suspension at a concentration of $10 \mathrm{~g} / \mathrm{L}, \mathrm{BSA}$ at a concentration of $100 \mathrm{~g} / \mathrm{L}$, and FITC-D in a concentration of $50 \mathrm{~g} / \mathrm{L}$. For mixtures of phospholipids and cholesterol the molar ratio amounted to 60:40. These aqueous media containing dissolved model active ingredients and dispersed lipids were used as the aqueous phase for the generation of $\mathrm{w} / \mathrm{fc}$ nano-emulsions as the next step of liposome production. 


\subsection{Preparation of $w / f_{c}$ Nanoemulsions and Liposomes}

The $\mathrm{w} / \mathrm{fc}$ nano-emulsion contained 1\% dispersed phase (aqueous lipid stock suspension, see above) and was used to produce liposomes via centrifugation. The hydrophobic phase consisted of the fluorocarbon perfluoro-perhydro-phenanthrene $\left(\mathrm{C}_{14} \mathrm{~F}_{24}\right)$, if not stated otherwise. The droplet size averaged around $180 \mathrm{~nm}$ and was found to be suitable for liposome production [18].

Liposomes were prepared by transferring the water droplets of the $\mathrm{w} / \mathrm{fc}_{\mathrm{c}}$ nanoemulsion containing the model API to a second aqueous phase via centrifugation (Figure 1).

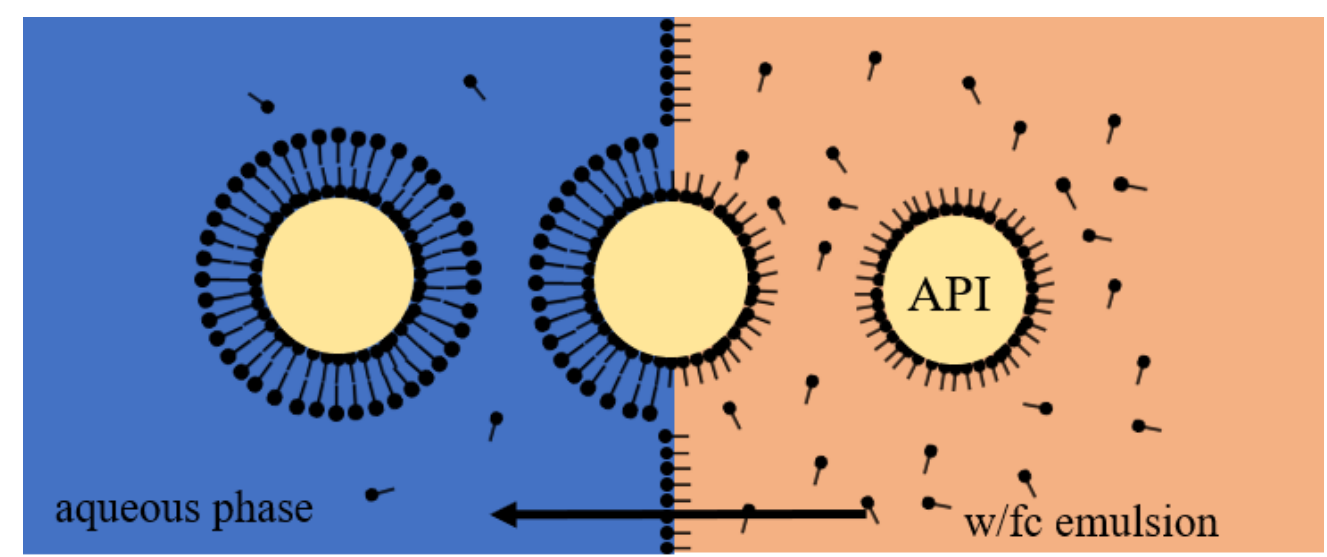

Figure 1. Schematic illustration of the centrifugation step to produce liposomes. Water droplets of a $\mathrm{w} / \mathrm{fc}$ nano-emulsion containing the model API ascend towards a second aqueous phase via centrifugation. During the transfer, a second outer lipid leaflet forms the liposome by covering the transferred emulsion droplet. Adapted from [18], Published by Advanced Materials Interfaces, 2020.

Thus, the lipid-coated aqueous cores of the nano-emulsion became surrounded by outer lipid leaflets, incorporating the API. The centrifugation step was performed at $0{ }^{\circ} \mathrm{C}$ and $4000 \times g$ for $30 \mathrm{~min}$ (Eppendorf Centrifuge $5430 \mathrm{R}$, Eppendorf, Hamburg, Germany). Because of the higher density of the surrounding fluorocarbon, the lighter water droplets ascend to the upper aqueous phase. A photograph of the transfer including FS as a hydrophilic marker is depicted in Figure 2a. The produced liposomes show an average size of $60 \mathrm{~nm}$, based on dynamic light scattering and small angle x-ray scattering measurements. A transmission electron microscopy (TEM) picture of DPPC-liposomes $(150 \mathrm{mM})$ evidences the production of vesicles (Figure 2b, TEM CM12, co. Philips, The Netherlands). Zeta potential of liposomes was measured at a constant voltage of $50 \mathrm{mV}$ with the Zetasizer Nano ZS (Malvern Instruments, Worcestershire, UK). For a detailed description of nanoemulsion and liposome preparation, as well as results of droplet and liposome size, please refer to Ullmann et al. [18].

\subsection{Detection Method}

For the detection of the model APIs FS, BSA and FITC-D inside the droplets, UV-Vis spectroscopy (DH-2000 Ocean Optics, Largo, FL, USA and UV-1900 Shimadzu, Kyoto, Japan) was chosen. The spectra of the initial phases-the fluorocarbon, the buffer, the emulsion, the stock solution and the diluted solution of either FS, BSA or FITC-D-were recorded beforehand. The encapsulated model API FS shows a significant absorption peak at $491 \mathrm{~nm}$, BSA at $280 \mathrm{~nm}$ and FITC-D at $493 \mathrm{~nm}$. The absorption spectra of different substances applied during the centrifugation process reveal that neither the fluorocarbon, the phosphate buffer, nor different DPPC concentrations of stock solutions expose a significant peak at the same position. PLs absorb at a wavelength of $230 \mathrm{~nm}$.

As an example, the spectra of different compounds needed for the preparation of liposomes are shown in Figure 3a. A comparison between the absorption spectrum of an emulsion with FS and an emulsion without FS reveals that the fluorocarbon has a shielding 
effect on the dye. However, detection, as well as distinction, is possible as there is a small peak visible at $458 \mathrm{~nm}$ for the emulsion, including FS.

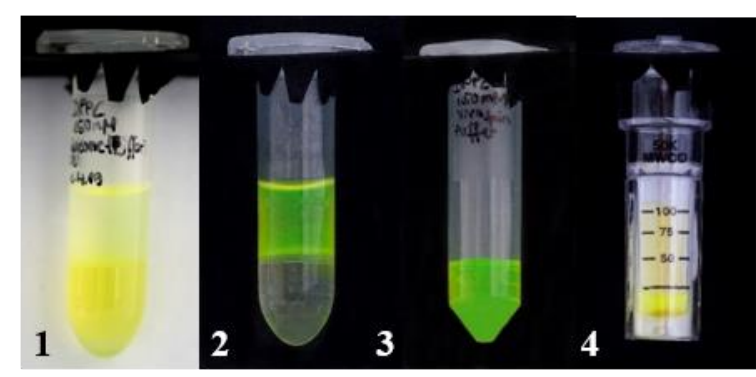

(a)

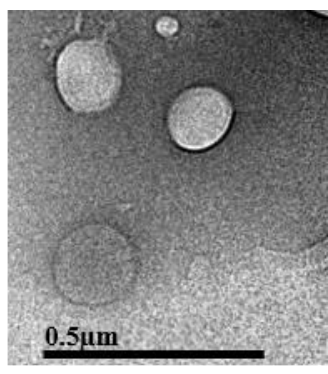

(b)

Figure 2. (a) Photographs of the centrifugation process performed in micro-reaction tubes. $1 . \mathrm{w} / \mathrm{fc}$ emulsion containing FS with phosphate buffer as the aqueous phase on top. The yellow color on top is due to the reflection of the lower phase. 2. Complete transfer of the droplets to the upper aqueous phase. 3. Filtrate after centrifugation with the Vivaspin ${ }^{\circledR}$. 4. Retentate (liposomes) left on the membrane of the Vivaspin ${ }^{\circledR}$. (b) TEM picture of DPPC-liposomes (150 mM). Negative staining of the aqueous phase surrounding the liposomes was performed by ammonium molybdate.

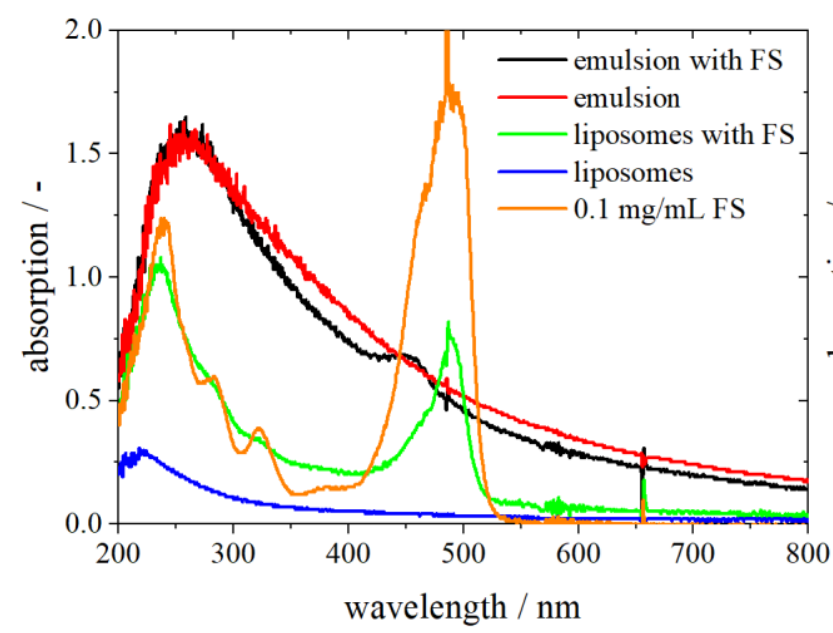

(a)

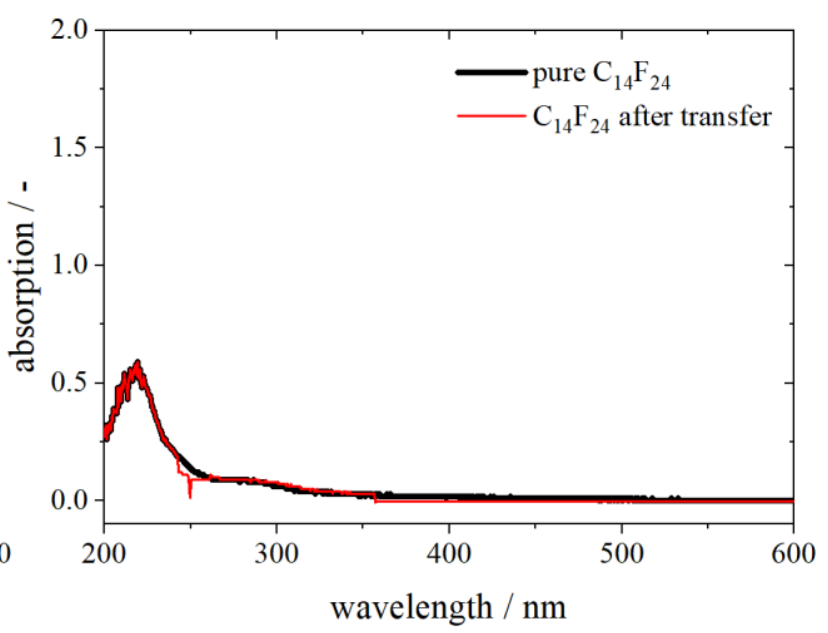

(b)

Figure 3. Absorption spectra of different compounds needed for the preparation of liposomes: (a) an emulsion with and without FS, liposomes prepared with and without FS and a pure stock solution of $0.1 \mathrm{mg} / \mathrm{mL}$ FS; (b) the spectrum of the pure fluorocarbon $\mathrm{C}_{14} \mathrm{~F}_{24}$ and after the transfer of emulsion droplets.

After centrifuging the $\mathrm{w} / \mathrm{fc}$ nano-emulsion and transferring of droplets to the upper aqueous phase, the lower hydrophobic phase $\left(\mathrm{C}_{14} \mathrm{~F}_{24}\right)$ was measured again. The fluorocarbon shows the same absorbance after centrifugation as in its pure condition, indicating that no FS nor phospholipids remain in the hydrophobic phase and the transfer is completed entirely (Figure 3b).

\subsection{Encapsulation Efficiency}

To determine the encapsulation efficiency of the produced liposomes, FS was added at a concentration of $10 \mathrm{~g} / \mathrm{L}$ to the lipid stock suspension, BSA at a concentration of $100 \mathrm{~g} / \mathrm{L}$ and FITC-D at a concentration of $50 \mathrm{~g} / \mathrm{L}$. The final concentration of the model APIs in the nano-emulsion was 1:100 of the initial concentration as the emulsions were prepared with $1 \%(v / v)$ of the dispersed phase.

After producing the nano-emulsion and centrifuging the droplets through the interface, the upper aqueous phase containing the liposomes and the model API is collected, and 
non-encapsulated FS is separated by Vivaspin $500^{\circledR}$ (Sartorius, Göttingen, Germany). The Vivaspin $500^{\circledR}$ has a capacity of $500 \mu \mathrm{L}$ and a separation limit of $50 \mathrm{kDa}$. For BSA and FITC-D, a Vivaspin $2^{\circledR}$ with a $100 \mathrm{kDa}$ cut off was chosen. To separate liposomes from free FS, BSA, or FITC-D, the samples were centrifuged for $3 \mathrm{~h}$ with $4000 \times g$ at $0{ }^{\circ} \mathrm{C}$. Liposomes remain in the filter module (retentate) while the non-encapsulated model API passes through the membrane and is quantified via the UV-Vis spectrometer (filtrate).

The encapsulation efficiency considering the initial concentration $\mathrm{c}_{0}$ is called $\mathrm{EE}_{\text {theor }}$ and its value is provided in $\%$ and is determined by

$$
\mathrm{EE}_{\text {theo }}(\%)=\left(1-\frac{\mathrm{c}_{\mathrm{F}}}{\mathrm{c}_{0}}\right) \cdot 100
$$

where $c_{F}$ is the amount of free model API in the filtrate and $c_{0}$ is the initial concentration of API in the emulsion.

\subsection{Recovery Rate (RR)}

In addition to the measurement of the free model API after the separation step, the amount of the marker in the complete aqueous phase was detected, e.g., shown here for FS $\left(\mathrm{RR}_{\mathrm{FS}}\right)$. Perchloric acid destroys the liposomes and allows the measurement of the total amount of FS which should add up to $100 \%$ of the initial amount. To determine the total amount of FS compared to the initial amount, the liposome suspension was treated with $70 \%$ perchloric acid at a volume ratio of 1:1 for solubilisation of liposomes and quantified via UV-Vis spectroscopy at $435 \mathrm{~nm}$ according to a calibration curve with perchloric acid, respectively. Treatment took place for $10 \mathrm{~min}$ and $50{ }^{\circ} \mathrm{C}$ on a heated shaker. In addition, possible losses of FS at the wall of the reaction tube were taken into account by washing the tubes with perchloric acid and quantification by UV-Vis spectroscopy. The retentate (liposome remains on the Vivaspin membrane) was treated likewise with perchloric acid to specify the amount of FS. The quantification is summed up as follows and was performed for FS, dextran and phospholipids:

$$
\operatorname{RR}_{\mathrm{x}}(\%)=\left(\frac{c_{x, \text { total }}}{\mathrm{c}_{0}}\right) \cdot 100
$$

where the index $x$ stands for either FS, D (dextran) or PL (phospholipid), $c_{x, \text { total }}$ is the total amount of model API or phospholipids found in the upper aqueous phase after transfer, and $c_{0}$ is the initial amount from the stock suspension. When referring to RR as a concentration, e.g., the RR of FS, it is defined as $c_{R R}$ in $\mathrm{mM}$.

\subsection{Encapsulation under Different Osmotic Conditions}

The efficiency of the encapsulation was monitored under different osmotic conditions inside and outside the liposomes. For this purpose, the phosphate buffer concentration was changed from $15 \mathrm{mM}$ to a range between $83 \mathrm{mM}$ and $250 \mathrm{mM}$, and no salt ions $(0 \mathrm{mM})$.

\subsection{Determination of Phospholipids}

The concentration of phospholipids in the filtrate as well as possible losses and the concentration of phospholipids that are completely transferred were determined by phosphorus assay according to Fiske [19]. Perchloric acid (70\%) destroys the phospholipid molecules while ascorbic acid and ammonium molybdate lead to a change of blue color under the presence of phosphorus.

\section{Results and Discussion}

\subsection{Loss of Phospholipids}

The losses of phospholipids during the process were monitored for the lipid DPPC at a concentration of 150 and $300 \mathrm{mM}$. It was found that a loss appears during sonication and that the stock suspension does not fully retain the initial concentration (cf. Table 1. (A) 
and (B)). Most phospholipids are found in the upper aqueous phase after centrifugation (cf. column (C)), as expected. In addition, small amounts remain on the wall of the reaction tube after the hydrophobic phase is removed (cf. column (D)). In that case the reaction tube was washed with the same volume of perchloric acid as the initial emulsion. In total, a loss of $30 \%$ is common for all phospholipids (cf. column (F)), which is mainly due to the greater amount of phospholipids that remain at the interface and cannot be completely collected.

Table 1. Concentration of phospholipids found in the initial stock suspension (A), the measured phospholipid amount in \% based on the initial amount (B), the amount found in the re-dispersed upper aqueous phase after centrifugation (C) and at the wall of the reaction tube after washing with the same volume of perchloric acid as the previous emulsion (D). (E) displays the sum of (C) and (D) while (F) is the total amount of phospholipids found after centrifugation based on (A) and equals the $R_{P L}$ according to Equation (2).

\begin{tabular}{lcccccc}
\hline Phospholipid & $(\mathrm{A}) / \mathbf{m M}$ & $\mathbf{( B ) / \%}$ & $\mathbf{( C ) / \mathbf { m M }}$ & $\mathbf{( D ) / m M}$ & $\mathbf{( E ) / m M}$ & $\mathbf{( F ) / \%}$ \\
\hline $150 \mathrm{mM}$ DPPC & 108.72 & 72.48 & 0.65 & 0.17 & 0.82 & 75.58 \\
$300 \mathrm{mM} \mathrm{DPPC}$ & 215.58 & 71.86 & 1.11 & 0.33 & 1.44 & 66.72 \\
\hline
\end{tabular}

\subsection{Recovery Rate}

For comparison, a stock suspension with FS was treated in the same way as a liposome suspension. Both absorption spectra show the same results after being heated up with perchloric acid, proving the completeness of FS release from liposomes and the consistency of the method. DPPG shows a bigger loss of FS during the liposome production process $\left(\mathrm{RR}_{\mathrm{FS}}\right.$ of $81 \%$ ) while the $\mathrm{RR}_{\mathrm{FS}}$ for liposomes produced with DPPC (with both 150 and $300 \mathrm{mM}$ ) is at $91 \%$ (Table 2). However, a concentration of $50 \mathrm{mM}$ DPPC in the stock suspension only leads to a $\mathrm{RR}_{\mathrm{FS}}$ of $48 \%$. A concentration of only $50 \mathrm{mM}$ was found to be too low for an adequate stabilization of $\mathrm{w} / \mathrm{fc}$ nano-emulsions (cf. Ullmann et al. [18]). The phospholipid DMPC shows a similar low $\mathrm{RR}_{\mathrm{FS}}$ of $45 \%$, even at $150 \mathrm{mM}$. Nonetheless, taken into account that losses during sonication and transfer with a high surface to volume ratio are to be considered, an $\mathrm{RR}_{\mathrm{FS}}$ of $91 \%$ is an unexpected high yield.

Table 2. Total amount of FS found after centrifugation and determined by applying perchloric acid to the aqueous phase. The recovery rate $R_{\mathrm{FS}}$ is calculated from Equation (2) for different phospholipids and concentrations.

\begin{tabular}{cc}
\hline Concentration, Phospholipid & $\mathbf{R R}_{\mathbf{F S}} / \mathbf{\%}$ \\
\hline 50 mM DPPC & 48.13 \\
150 mM DPPC & 91.97 \\
300 mM DPPC & 91.46 \\
150 mM DMPC & 45.08 \\
150 mM DPPG & 81.26 \\
300 mM DPPG & 81.51 \\
\hline
\end{tabular}

For the depiction of data regarding the $\mathrm{EE}$, the theoretical value $\mathrm{E}_{\text {theo }}$ was replaced with a corrected efficiency $\mathrm{EE}_{\mathrm{cor}}$ based on the FS-concentration $\mathrm{C}_{\mathrm{RR}}$ (or other model APIs, respectively) found after the transfer:

$$
E_{c o r}(\%)=\left(1-\frac{c_{F}}{c_{R R}}\right) \cdot 100
$$

\subsection{Effect of Different Fluorocarbons on Encapsulation Efficiency}

Different fluorocarbons were examined regarding their contribution to encapsulate the model API FS, namely perfluoro-heptane $\left(\mathrm{C}_{7} \mathrm{~F}_{16}\right)$, perfluoro-1,3-dimeth ylcyclohexane $\left(\mathrm{C}_{8} \mathrm{~F}_{16}\right)$ and perfluoro-methyl-decalin $\left(\mathrm{C}_{11} \mathrm{~F}_{20}\right)$ and perfluoro-perhydrophenanthrene $\left(\mathrm{C}_{14} \mathrm{~F}_{24}\right)$ (Figure 4$)$. For a better comparison and classification, two different calculation methods were considered. $\mathrm{EE}_{\text {theo }}$ as in Equation (1) is based 
on the initial amount of FS while $\mathrm{EE}_{\mathrm{cor}}$ (Equation (3)) uses the values of the RR for calculation. The latter takes losses of the API into account. Hence, a large difference between $\mathrm{EE}_{\text {theo }}$ and $\mathrm{EE}_{\mathrm{cor}}$ demonstrates a poor encapsulation. The results reveal a significant distinction between $\mathrm{EE}_{\text {theo }}$ and $\mathrm{EE}_{\mathrm{cor}}$ for the fluorocarbons $\mathrm{C}_{7} \mathrm{~F}_{16}$ and $\mathrm{C}_{8} \mathrm{~F}_{16}$. While for $\mathrm{C}_{11} \mathrm{~F}_{20}$ the losses of $\mathrm{FS}$ are smaller, visual observations during the experiment showed an unstable nano-emulsion with droplets quickly moving to the upper phase without centrifugation. Figure 4 leads to the conclusion that the lower the density of the fluorocarbon phase the lower the encapsulation efficiency because losses are higher. The instability of nano-emulsions prepared with lighter fluorocarbons according to their molecular weight has been reported previously [20]. Thus, a higher loss of API is likely if the stability of nano-emulsions is already poor. As $\mathrm{C}_{14} \mathrm{~F}_{24}$ shows the best results, it was applied for further experiments.

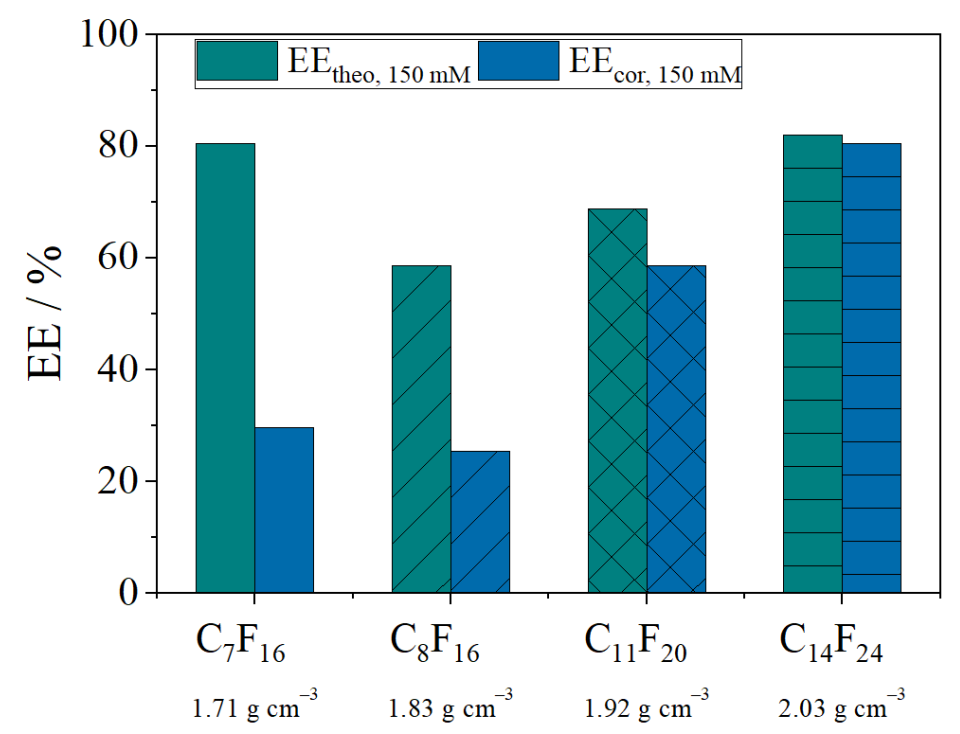

Figure 4. Effect of different fluorocarbons (perfluoro-heptane $\left(\mathrm{C}_{7} \mathrm{~F}_{16}\right)$, perfluoro-1,3-dimethylcyclohexane $\left(\mathrm{C}_{8} \mathrm{~F}_{16}\right)$, perfluoro-methyl-decalin $\left(\mathrm{C}_{11} \mathrm{~F}_{20}\right)$ and perfluoro-perhydro-phenanthrene $\left.\left(\mathrm{C}_{14} \mathrm{~F}_{24}\right)\right)$ on the encapsulation of FS with a lipid stock suspension of $150 \mathrm{mM}$ DPPC. The green bars show the $\mathrm{EE}_{\text {theo }}$ based on the initial amount of FS added to the stock suspension (Equation (1)), while blue bars depict the $\mathrm{EE}_{\text {cor }}$ (Equation (3)), based on the RR (Equation (2)). In addition, the densities of each fluorocarbon are listed.

\subsection{Influence of Different DPPC Concentrations and the Addition of Cholesterol}

A separation of free and encapsulated FS is indispensable and was carried out in an additional experimental set up with a Vivaspin $500^{\circledR}$, exemplarily for the phospholipid DPPC with a concentration of $150 \mathrm{mM}$ in $\mathrm{ddH}_{2} \mathrm{O}$. The absorption spectrum of the filtrate containing the free FS compares to the spectrum of the diluted stock solution of FS and allows the determination of the encapsulation efficiency. In addition, the retentate was treated with perchloric acid to solubilise the liposomes from the Vivaspin ${ }^{\circledR}$ membrane. For best results, the membrane was washed twice with the same solution. The encapsulation efficiency calculated with different methods is shown in Figure 5 . The $\mathrm{RR}_{\mathrm{FS}}$ is almost $100 \%$ in comparison to the initial concentration (97\%). As the filtrate only contains small amounts of $\mathrm{FS}$ and the $\mathrm{RR}_{\mathrm{FS}}$ is high, both the $\mathrm{EE}_{\text {theo }}$ and $\mathrm{EE}_{\mathrm{cor}}$ equal $99 \%$. The retentate left on the membrane of the Vivaspin ${ }^{\circledR}$ measures $92 \%$ (calculated as in Equation (3)) which differs by $8 \%$ from the EE calculated from the filtrate. Thus, in the following depictions, results are based on the filtrate. 


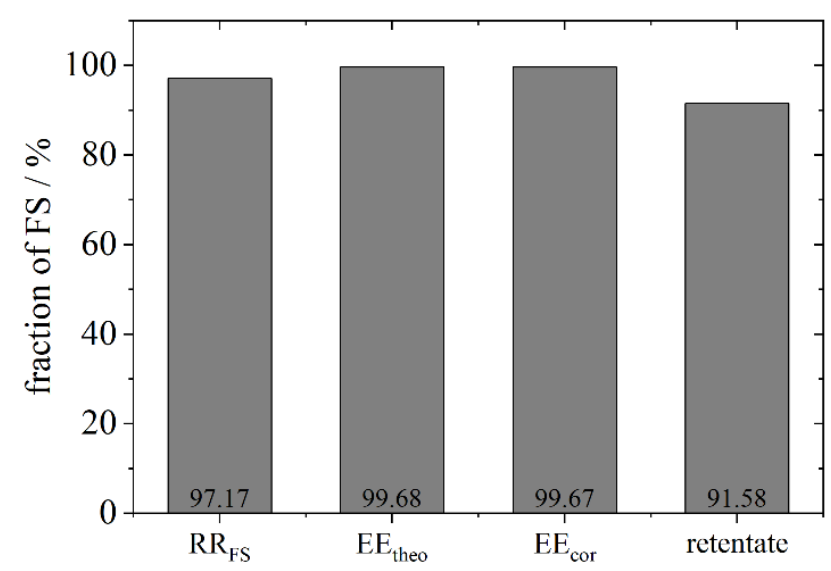

Figure 5. Depiction of different fractions shown for FS for the phospholipid DPPC with a concentration in the stock suspension of $150 \mathrm{mM}$. Depicted are the recovery rate $\mathrm{RR}_{\mathrm{FS}}$ calculated according to Equation (2), the encapsulation efficiency $\mathrm{EE}_{\text {theo }}$ calculated according to Equation (1), the encapsulation efficiency $\mathrm{EE}_{\text {cor }}$ calculated according to Equation (3) and the retentate (calculated as in Equation (3)) after treatment of the Vivaspin ${ }^{\circledR}$ membrane with perchloric acid.

In the following, the encapsulation efficiency of liposomes produced from different phospholipid concentrations and with the addition of cholesterol were examined (Table 3). Different amounts of DPPC in the stock solution are able to encapsulate different amounts of FS: a high concentration of $300 \mathrm{mM}$ leads to an encapsulation efficiency of $84 \%$, calculated from Equation (3), whereas only $50 \mathrm{mM}$ of PL in the stock solution encapsulate a maximum amount of $48 \%$. This leads to the conclusion that higher concentrations of PL used for the production of liposomes encapsulate a greater amount of the model API because of a more stable vesicle. In addition, the losses detected with the phosphorus assay explain that, for a stabilization of water droplets, a greater amount of phospholipids is necessary.

Table 3. Encapsulation efficiency $E_{\mathrm{cor}}$ according to Equation (3) of different phospholipids and concentrations and the zeta potential $\zeta$ in $\mathrm{mV}$.

\begin{tabular}{ccc}
\hline Concentration, Phospholipid & EE $_{\text {cor }} / \%$ & $\zeta$ Potential/mV \\
\hline 50 mM DPPC & 48.1 & \\
150 mM DPPC & 80.4 & $-2.4 \pm 0.5$ \\
300 mM DPPC & 83.6 & $-3.4 \pm 0.6$ \\
300 mM DPPC + Cholesterol (60:40) & 73.7 & $-44.4 \pm 1.0$ \\
150 mM DMPC & 96.8 & 63.1 \\
\hline
\end{tabular}

The use of a phospholipid with a shorter chain length (DMPC) shows similar results: a shorter chain length leads to an EE of $96 \%$. However, the $\mathrm{RR}_{\mathrm{FS}}$ is much lower (by $40 \%$ ) in comparison to DPPC (cf. Table 2) indicating a bigger loss during the process.

In general, the addition of cholesterol or negatively charged phospholipid head groups stabilize membranes and liposomes. Hence, a better encapsulation efficiency can be hypothesized. This hypothesis was tested by encapsulating FS with pure DPPG as well as a mixture of DPPC and cholesterol (60:40 mol-\%) in comparison to pure DPPC. The filtrate was measured with the UV-Vis. The absorption of the filtrate with DPPG- and DPPC+chol stabilized liposomes is much higher than the measured absorption of the filtrate from DPPC liposomes. Thus, the encapsulation efficiency differs accordingly from $83 \%$ for pure DPPC liposomes to 74\% when cholesterol is added. Ahmad et al. [21] achieve an encapsulation efficiency of $25.86 \%$ for a mixture of PC and chol of $0.9: 1$, which is increased to $42.34 \%$ with a higher PC proportion of 1.1:1. A decrease of the encapsulation efficiency with an increase of the cholesterol amount was also described by Briguila et al. [22]. As the transition temperature is not elevated, but cholesterol increases the fluidity of the 
membrane even below the transition temperature, this leads to a higher bending modulus (or bilayer toughness) which is likely to be the explanation for the lower encapsulation efficiency [23]. Cholesterol changes the characteristics of the membrane and thus, is likely to slow down the dynamics of membrane fusion. Chernomordik et al. describe membrane fusion as a function of the membrane characteristics such as the density of fusion proteins and the lipid composition [24]. Two membranes come into contact and form a hemi-fusion before emerging together. The liposome formation is the opposite process: an emulsion droplet contacts the interface and the bilayer is formed while passing the interface. At the moment of detachment of the newly formed liposome from the interface, they are not yet fully closed leading to a leakage of the model API. The rapidity of closure after the detachment defines the encapsulation efficiency. As cholesterol increases the bending modulus, as also described by Pan et al. and Needham et al., membranes need more time to close after detachment from the interface and, thus, lose more of the model API $[25,26]$.

Likewise, liposomes produced by the anionic phospholipid DPPG show a smaller encapsulation efficiency of $63 \%$ in comparison to liposomes formed by emulsion droplets stabilized with the zwitterionic DPPC. Jing et al. [27] observe a reduction of the lipid bilayer thickness and density due to higher electron net charge density. This phenomenon can be attributed to the negatively charged headgroups of DPPG whose repelling force is stronger than that of the zwitterionic DPPC molecules. Thus, the distance between headgroups and the inclination angle increases. Additionally, the membrane rigidity increases, as described by Faizi et al. [28]. It is assumed that the bilayer is more permeable and FS is released more easily. These results are in accordance with the $\mathrm{RR}_{\mathrm{FS}}$ for DPPG which also showed more losses.

Zeta potential measurements show a small negative potential for the phospholipids DPPC and DPPC + chol. and a larger negative potential for the negatively charged phospholipid DPPG. We assume that the bending modulus has a stronger influence on the encapsulation than the zeta potential. Nonetheless, for future leakage studies, zeta potential measurements should be taken into account in more detail for different liposome formulations and encapsulated APIs.

\subsection{Encapsulation Efficiency of BSA and Fluorescently Labelled Dextran under Different Osmotic Conditions}

Besides the inquiry regarding the encapsulation of a low molecular weight dye, the question arises as to whether high molecular weight proteins such as BSA show similar results. In addition, future application makes it necessary to investigate the influence of different osmotic conditions that may be present in the blood outside the liposome. Two different set-ups were applied: the phosphate concentration inside the liposome was kept constant at $83 \mathrm{mM}$ while the outer aqueous phase changed from $83 \mathrm{mM}$ to $250 \mathrm{mM}$; and in a second experiment, the influx and efflux were examined. The influx describes an osmotic situation where a lower salt concentration outside, in comparison to the inside of the liposome, leads to a motion of water ions into the vesicle through the membrane. An efflux is the opposite effect: due to a higher osmotic pressure inside the liposome, the ions move from the inside to the outside. Both cases were performed by creating a maximum difference in salt concentrations ( $0 \mathrm{mM}$ and $250 \mathrm{mM}$ ); additionally, a comparison was implemented with the same conditions inside and outside the liposome.

It is expected that the higher the outside salt concentration, the bigger the efflux and, thus, leaking of the model API. These assumptions are reflected by the results shown in Figure 6. In comparison to FS, the EE of BSA is slightly lower. As the molecular weight is much higher, an encapsulation appears more difficult and thus, a lower EE was expected. However, with the same osmotic conditions inside and outside the liposome $(83 \mathrm{mM}$ $\mathrm{PB})$, an EE of $78 \%$ can be achieved, which is still higher than efficiencies reported in the literature. The higher the salt concentration outside the liposome and thus, the efflux, the lower the EE. For a maximum difference of salt ions between the inside and outside, an EE of $66 \%$ is detected. 


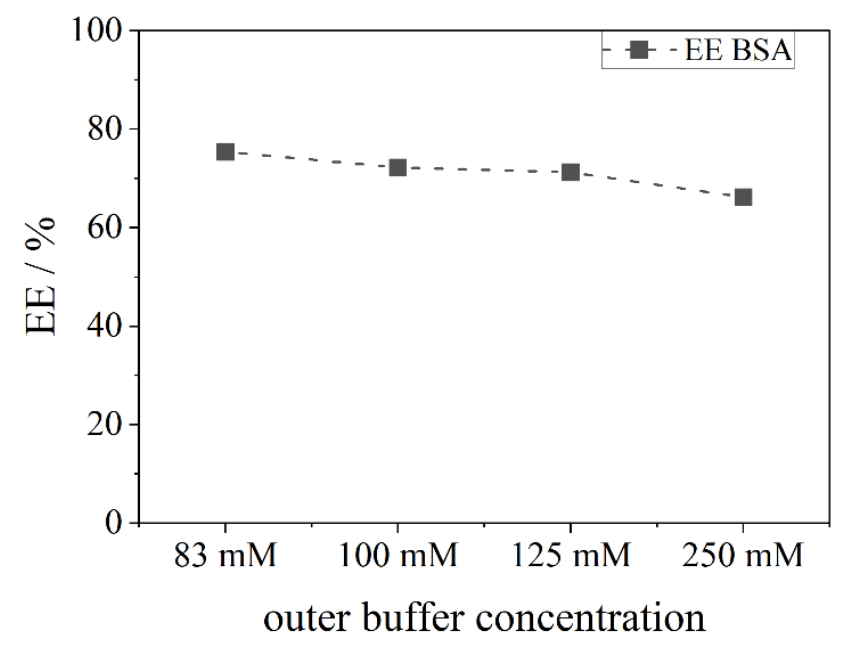

Figure 6. Encapsulation efficiency (EE) of BSA in liposomes prepared from a $150 \mathrm{mM}$ DPPC stock suspension. The inner buffer concentration remains at $83 \mathrm{mM}$ of phosphate buffer, while the outer buffer concentration (the upper aqueous phase) changes from $83 \mathrm{mM}$ to $250 \mathrm{mM}$.

Furthermore, FITC-D was investigated regarding the EE (Figure 7). A similar result is also observed here. While no salt $(0 \mathrm{mM})$ achieves the highest EE $(98 \%)$, an efflux reduces it by $30 \%$. In comparison, high salt concentrations $(250 \mathrm{mM})$ encapsulate less dextran, but an influx stabilizes the liposome and results in a better EE (95\%). These results indicate that, in general, ultrapure water inside and outside the liposome leads to less interference of phospholipids and more stability whereas an efflux destabilizes the lipid vesicles. An influx increases the stability in comparison to the efflux which was not expected, as a strong influx could lead to bursting of liposomes and, thus, set free more of the API.

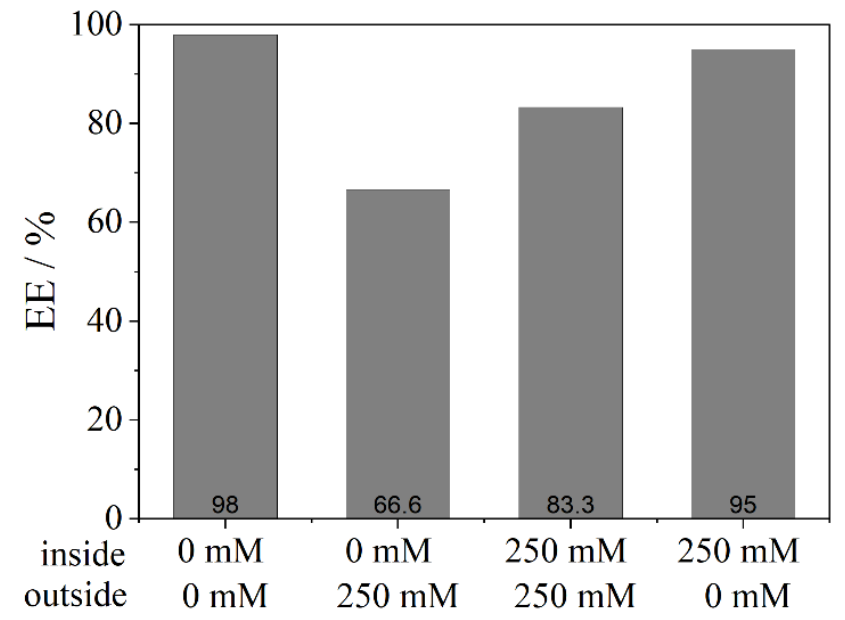

Figure 7. Encapsulation efficiency (EE) of FITC-D mimicking efflux and influx: The first line on the $\mathrm{x}$-axis shows the concentration of phosphate buffer at the inside of the liposome, while the second line equals the concentration of phosphate buffer at the outside aqueous phase after centrifugation. Liposomes were prepared from a $150 \mathrm{mM}$ DPPC stock suspension.

\section{Conclusions}

The encapsulation not only of low molecular weight substances (FS), but also of macromolecules (BSA and dextrane) with $>60 \mathrm{kDa}$ and without a high affinity to the bilayer, which could enhance the encapsulation, was successfully achieved. The encapsulation efficiency was determined and found to be between $48-98 \%$ for the fluorocarbon $\mathrm{C}_{14} \mathrm{~F}_{24}$ depending on the used phospholipid. Other fluorocarbons showed a lower EE (20-60\%) which revealed that not all fluorocarbons encapsulate the model APIs equally well. A 
higher density of the fluorocarbon is to be favoured for the process. These achievements were possible due to the novel preparation method of liposomes by using a centrifuge to transfer droplets from a $\mathrm{w} / \mathrm{fc}$ nano-emulsion. The results show that compounds which stabilize a membrane, such as cholesterol or charged phospholipids, have a negative effect on the encapsulation efficiency. This effect is likely to be due to the greater bending elasticity of the membrane which leads to a slower closure of the vesicle after the detachment of the liposome from the interface. Hence, the leakage increases. For further stabilization of liposomes and for mimicking a future application in the blood, osmotic conditions were imitated. An efflux negatively affects the encapsulation efficiency, an influx leads to a better encapsulation than under efflux conditions, while best results were found for pure water inside and outside.

Thus, the results and the liposome preparation method show not only the capacity for large-scale application, but the method of analysis is also applicable for a scale-up. These data demonstrate a promising alternative for industry in producing liposomes for pharmaceutical application, which additionally hold the capacity to encapsulate high amounts of macromolecules.

Author Contributions: Conceptualization, K.U.; methodology, K.U.; validation, K.U.; formal analysis, K.U.; investigation, K.U.; resources, K.U.; data curation, K.U.; writing-original draft preparation, K.U.; writing-review and editing, G.L.; visualization, K.U.; supervision, G.L. and H.N.; project administration, G.L., H.N.; funding acquisition, H.N. All authors have read and agreed to the published version of the manuscript.

Funding: This research was funded by the Phospholipid Research Center, grant number HNI2019-072/2-1.

Institutional Review Board Statement: Not applicable.

Informed Consent Statement: Not applicable.

Data Availability Statement: Not applicable.

Acknowledgments: We sincerely thank Lipoid (Ludwigshafen) for donating phospholipids for this research and Barbara Miranda for the TEM imaging of liposomes. We acknowledge support by the KIT-Publication Fund of the Karlsruhe Institute of Technology.

Conflicts of Interest: The authors declare no conflict of interest.

\section{References}

1. Siegel, R.L.; Miller, K.D.; Jemal, A. Cancer statistics, 2019. CA Cancer J. Clin. 2019, 69, 7-34. [CrossRef]

2. Peer, D.; Karp, J.M.; Hong, S.; Farokhzad, O.C.; Margalit, R.; Langer, R. Nanocarriers as an emerging platform for cancer therapy. Nat. Nanotech. 2007, 2, 751-760. [CrossRef]

3. Bayyurt, B.; Tincer, G.; Almacioglu, K.; Alpdundar, E.; Gursel, M.; Gursel, I. Encapsulation of two different TLR ligands into liposomes confer protective immunity and prevent tumor development. J. Control. Release 2017, 247, 134-144. [CrossRef]

4. van Hoogevest, P.; Wendel, A. The use of natural and synthetic phospholipids as pharmaceutical excipients. Eur. J. Lipid Sci. Technol. 2014, 116, 1088-1107. [CrossRef]

5. Akashi, K.; Miyata, H.; Itoh, H.; Kinosita, K. Preparation of giant liposomes in physiological conditions and their characterization under an optical microscope. Biophys. J. 1996, 71, 3242-3250. [CrossRef]

6. Szoka, F.; Papahadjopoulos, D. Procedure for preparation of liposomes with large internal aqueous space and high capture by reverse-phase evaporation. Proc. Natl. Acad. Sci. USA 1978, 75, 4194-4198. [CrossRef] [PubMed]

7. Popa, R.; Vrânceanu, M.; Nikolaus, S.; Nirschl, H.; Leneweit, G. Entrance Effects at Nanopores of Nanocapsules Functionalized with Poly(ethylene glycol) and Their Flow through Nanochannels. Langmuir 2008, 24, 13030-13036. [CrossRef] [PubMed]

8. Olson, F.; Hunt, C.A.; Szoka, F.C.; Vail, W.J.; Papahadjopoulos, D. Preparation of liposomes of defined size distribution by extrusion through polycarbonate membranes. Biochim. Biophys. Acta. Biomembr. 1979, 557, 9-23. [CrossRef]

9. Brandl, M.; Bachmann, D.; Drechsler, M.; Bauer, K.H. Liposome Preparation by a New High Pressure Homogenizer Gaulin Micron Lab 40. Drug. Dev. Ind. Pharm. 1990, 16, 2167-2191. [CrossRef]

10. Cornell, B.A.; Middlehurst, J.; Separovic, F. Small unilamellar phospholipid vesicles and the theories of membrane formation. Faraday Discuss. Chem. Soc. 1986, 81, 163-178. [CrossRef]

11. Cornell, B.A.; Fletcher, G.C.; Middlehurst, J.; Separovic, F. The lower limit to the size of small sonicated phospholipid vesicles. Biochim. Biophys. Acta. Biomembr. 1982, 690, 15-19. [CrossRef] 
12. Colletier, J.-P.; Chaize, B.; Winterhalter, M.; Fournier, D. Protein encapsulation in liposomes: Efficiency depends on interactions between protein and phospholipid bilayer. BMC Biotechnol. 2002, 2, 9. [CrossRef]

13. Hwang, S.Y.; Kim, H.K.; Choo, J.; Seong, G.H.; Hien, T.B.D.; Lee, E.K. Effects of operating parameters on the efficiency of liposomal encapsulation of enzymes. Colloids Surf. B Biointerfaces 2012, 94, 296-303. [CrossRef] [PubMed]

14. Xu, X.; Costa, A.; Burgess, D.J. Protein encapsulation in unilamellar liposomes: High encapsulation efficiency and a novel technique to assess lipid-protein interaction. Pharm. Res. 2012, 29, 1919-1931. [CrossRef] [PubMed]

15. Kirby, C.J.; Gregoriadis, G. Preparation of liposomes containing factor VIII for oral treatment of haemophilia. J. Microencapsul. 1984, 1, 33-45. [CrossRef]

16. Pautot, S.; Frisken, B.J.; Weitz, D.A. Engineering asymmetric vesicles. Proc. Natl. Acad. Sci. USA 2003, 100, 10718-10721. [CrossRef]

17. de Matos, M.B.C.; Miranda, B.S.; Rizky Nuari, Y.; Storm, G.; Leneweit, G.; Schiffelers, R.M.; Kok, R.J. Liposomes with asymmetric bilayers produced from inverse emulsions for nucleic acid delivery. J. Drug Target. 2019, 27, 1-9. [CrossRef]

18. Ullmann, K.; Meier, M.; Benner, C.; Leneweit, G.; Nirschl, H. Water-in-Fluorocarbon Nanoemulsions Stabilized by Phospholipids and Characterized for Pharmaceutical Applications. Adv. Mater. Interfaces 2021, 8, 2001376. [CrossRef]

19. Fiske, C.H.; Subbarow, Y. The Colorimetric Determination of Phosphorus. J. Biol. Chem. 1925, 66, 375-400. [CrossRef]

20. Kabalnov, A.S.; Shchukin, E.D. Oswald Ripening Theory: Applications to Fluorocarbon Emulsion Stability. Adv. Colloid Interface Sci. 1992, 38, 69-97. [CrossRef]

21. Ahmad, I.; Arsalan, A.; Ali, S.A.; Sheraz, M.A.; Ahmed, S.; Anwar, Z.; Munir, I.; Shah, M.R. Formulation and stabilization of riboflavin in liposomal preparations. J. Photochem. Photobiol. B 2015, 153, 358-366. [CrossRef] [PubMed]

22. Briuglia, M.-L.; Rotella, C.; McFarlane, A.; Lamprou, D.A. Influence of cholesterol on liposome stability and on in vitro drug release. Drug. Deliv. Transl. Res. 2015, 5, 231-242. [CrossRef]

23. Coderch, L.; Fonollosa, J.; de Pera, M.; Estelrich, J.; La Maza, A.; de Parra, J. Influence of cholesterol on liposome fluidity by EPR: Relationship with percutaneous absorption. J. Control. Release 2000, 68, 85-95. [CrossRef]

24. Chernomordik, L.V.; Kozlov, M.M. Mechanics of membrane fusion. Nat. Struct. Mol. Biol. 2008, 15, 675-683. [CrossRef] [PubMed]

25. Pan, J.; Tristram-Nagle, S.; Nagle, J.F. Effect of cholesterol on structural and mechanical properties of membranes depends on lipid chain saturation. Phys. Rev. E Stat. Nonlin. Soft Matter Phys. 2009, 80, 21931. [CrossRef]

26. Needham, D.; Nunn, R.S. Elastic deformation and failure of lipid bilayer membranes containing cholesterol. Biophys. J. 1990, 58, 997-1009. [CrossRef]

27. Jing, H.Y.; Hong, D.H.; Kwak, B.D.; Choi, D.J.; Shin, K.; Yu, C.-J.; Kim, J.W.; Noh, D.Y.; Seo, Y.S. X-ray Reflectivity Study on the Structure and Phase Stability of Mixed Phospholipid Multilayers t. Langmuir 2009, 25, 4198-4202. [CrossRef] [PubMed]

28. Faizi, H.A.; Frey, S.L.; Steinkühler, J.; Dimova, R.; Vlahovska, P.M. Bending rigidity of charged lipid bilayer membranes. Soft Matter 2019, 15, 6006-6013. [CrossRef] [PubMed] 